\title{
VirtualTche - Mundo Imersivo do Instituto Federal Farroupilha - Campus Panambi
}

\author{
Rosana Wagner' ${ }^{2}$ Augusto Moura², Sirlei Rigodanzo Koslowski ${ }^{2}$, Liliana Maria \\ Passerino ${ }^{1}$, Sandra Dutra Piovesan ${ }^{1}$ \\ ${ }^{1}$ Programa de Pós-Graduação em Informática na Educação - Universidade Federal do \\ Rio Grande do Sul (UFRGS) \\ Avenida Paulo Gama, 110 - Prédio 12105 - $3^{\circ}$ andar sala 332 \\ Porto Alegre - RS - Brazil \\ ${ }^{2}$ Instituto Federal Farroupilha - Campus Panambi (IFFarroupilha) \\ Rua Erechim, 860 - Panambi - RS - Brasil \\ \{rosanawagner, lpasserino, sanpiovesan\}@gmail.com, sirlei@iffpb.edu.br, \\ augustomoura94@hotmail.com
}

\begin{abstract}
Information technology and communication through the use of software developed for specific environments has aided education through the pursuit of new technologies that provide students with the subject content more closely the real world, and in order to seek tools that will expanding current forms of learning, so that education has more dynamic aspects. This work was developed by the research group on Immersive Environments and aims at the development of the"'world" Instituto Federal Farroupilha and future use of this environment for teaching purposes, replacing the use of static environments such as traditional Moodle Virtual Learning Environments and TelEduc.
\end{abstract}

Resumo. As tecnologias da informação e comunicação, através do uso de softwares desenvolvidos para ambientes especificos tem auxiliado a educação através da busca de novas tecnologias que disponibilizam aos alunos os conteúdos disciplinares de forma mais próxima do mundo real, bem como no sentido de buscar ferramentas que venham a expandir as formas de aprendizagem atual, fazendo com que a educação tenha aspectos mais dinâmicos. Este trabalho foi desenvolvido pelo grupo de pesquisa sobre Ambientes Imersivos e tem como finalidade o desenvolvimento do "mundo" Instituto Federal Farroupilha e a futura utilização deste ambiente com fins pedagógicos, substituindo o uso de ambientes estáticos, como os tradicionais Ambientes Virtuais de Aprendizagem Moodle e TelEduc.

\section{Introdução}

A inclusão social ganhou força nas últimas décadas em todos os setores da sociedade e principalmente na educação (Beyer, 2005). A flexibilização curricular e a utilização de métodos individuais, assim como, ajuda pedagógica ajustada às possibilidades e necessidades de cada aluno devem ser possíveis (Beyer, 2005). Neste sentido, que as tecnologias de informação e comunicação através de Ambientes Virtuais Aprendizagens 
(AVA) podem ajudar a estabelecer ambientes mais adequados ao contexto dos estudantes (Passerino e Santarosa, 2006).

Recentemente surgiram experiências pedagógicas que têm sido realizadas em mundos virtuais, mais especificamente em mundos virtuais 3D on-line, que chamam a nossa atenção pelo grau de envolvimento e imersão dos estudantes com os conteúdos dos cursos, com os colegas e com o próprio professor, que não é reproduzido nos tradicionais AVAs: Moodle, Teleduc, Amadeus, etc. (Mattar, 2008) Em um ambiente virtual 3D, você vê o seu próprio avatar. Na informática, avatar é um cibercorpo inteiramente digital, uma figura gráfica de complexidade variada que empresta sua vida simulada para o transporte de cibernautas para dentro dos mundos paralelos do ciberespaço (Santaella, 2003). Você está situado, vê e ouve coisas daquele ponto de vista e então parece estar presente naquele ambiente (Mattar, 2008). Assim o estudante deixa de ser consumidor passivo do aprendizado, ou mesmo apenas criador de seu próprio conteúdo, para ser envolvido na criação de suas próprias atividades, suas experiências e de seu próprio ambiente de aprendizagem. (Mattar, 2008)

Este artigo tem por objetivo apresentar a modelagem, o desenvolvimento e uma proposta de aplicação de um ambiente imersivo possibilitando o ensino e a comunicação, através de um espaço que reproduza um ambiente próximo do mundo real, minimizando a distância dos tradicionais ambientes virtuais que geralmente são estáticos e impossibilitam uma interação mais concreta. Também é objetivo a apresentação das ferramentas selecionadas para esta modelagem.

O artigo está organizado como segue: no capítulo 2 é apresentada uma revisão bibliográfica sobre Realidade Virtual, AVAs tridimensionais e Ambientes Imersivos, também são apresentados os ambientes de realidade virtual Second Life e OpenSim, o capítulo 3 é dedicado a apresentação dos trabalhos relacionados. No quarto capitulo apresenta-se a metodologia de desenvolvimento do trabalho. $O$ quinto capítulo apresenta o "mundo" Instituto Federal Farroupilha - campus Panambi em 3D, juntamente com as soluções de desenvolvimento encontradas. $O$ sexto capítulo apresenta algumas considerações finais. O sétimo capítulo apresenta as referências bibliográficas que suportaram as pesquisas realizadas.

\section{Realidade Virtual, AVAS Tridimensionais e Ambientes Imersivos}

A evolução constante da tecnologia está levando à educação para novos caminhos, bem mais atrativa aos estudantes, tornando possível a utilização de novas ferramentas, levando a uma evolução no processo de ensino e aprendizagem. A Realidade Virtual ocupa um importante lugar nessa evolução.

O uso da Realidade Virtual (RV) na educação tende a promover um novo paradigma educacional, onde os estudantes não só recebem informações, mas também interagem entre si, manipulando conteúdo de diversas formas, manipulando o alvo que deve ser analisado, explorado e estudado (Matos, 2011). Uma das principais características da RV é a possibilidade de interação social, proporcionando a capacidade de ações e reações instantaneamente, em tempo real (Matos, 2011).

$\mathrm{Na}$ Realidade Virtual por imersão, o utilizador tem a sensação real de estar dentro do mundo virtual e que é capaz de manipular os objetos ali presentes como se 
eles fossem reais, visto que com o desenvolvimento tecnológico, estes objetos passaram a responder às interações realizadas pelo usuário (Braga, 2011).

Realidade virtual, realidade aumentada e suas variações representam técnicas de interface computacional que levam em conta o espaço tridimensional. Nesse espaço, o usuário atua de forma multissensorial, explorando aspectos deste espaço por meio da visão, audição e tato. Conforme a tecnologia disponível é possível também explorar o olfato e o paladar. Percepções corpóreas, como frio, calor e pressão, estão incluídas no tato, através da pele (Kirner, 2011).

Outros benefícios são observados com o uso de Realidade Virtual na educação. Segundo Clark (2006) a Realidade Virtual pode ser usada para tornar o aprendizado mais interessante e divertido com o objetivo de melhorar a motivação e a atenção, reduzindo custos quando a utilização do objeto e do ambiente real for mais dispendiosa que a simulação. Também possibilita que se explorem situações que são impossíveis de serem feitas no mundo real, por exemplo: explorar um planeta como Marte, viajar dentro do corpo humano, fazer explorações submarinas ou dentro de cavernas, visitar lugares muito pequenos para serem vistos (moléculas) ou muito caros ou muito distantes, ou ainda porque esse lugar está no passado (lugares históricos). Outro fator de destaque na RV é a melhoria de transferência de conhecimento, onde há a possibilidade de acessar o conteúdo de aprendizagem em qualquer lugar e em qualquer tempo (em caso de uso de Realidade Virtual na Internet), eliminando em casos de experimentos os riscos e perigos para o ambiente, para o professor ou para o aprendiz. Devido a esse último benefício, redução de riscos de acidentes, as simulações têm sido cada vez mais utilizadas em treinamento militar, na medicina e na aviação, em campos onde decisões e ações dos aprendizes podem causar danos ou mortes (Marins, 2007).

Em consonância com esta forma de utilização da Realidade Virtual na educação, está a Zona de Desenvolvimento Proximal proposta por Vygotsky (1991) que é a distância entre o nível de desenvolvimento real, que se costuma definir através da solução independente de problemas, e o nível de desenvolvimento potencial, determinado através da solução de problemas sob a orientação de um adulto ou em colaboração com companheiros mais capazes. Além disso, a interação entre alunos atende a perspectiva construtivista/interacionalista, pois os ambientes desenvolvidos, como o Second Life e o SLoodle, possibilitam a interação com companheiros mais capazes e resultam na formação de um sujeito autônomo e ativo na estruturação de seu conhecimento.

Ambientes imersivos são os espaços que utilizam sistemas computacionais para promover um estado de ilusão perceptiva e que incitam o visitante à participação. A imersão é usada como um estágio para aniquilar a diferença entre realidade e representação e como instrumento de persuasão da mente fazendo a passagem entre o realismo e o fantasioso (Medeiros, 2005).

As opções para uso, cada vez mais crescente de mundos virtuais na educação são diversas, podendo citar: Kaneva (http://www.kaneva.com/), HIPIHI (http://www.hipihi.com), IMVU (http://www.imvu.com), Metaplace (https://www.metaplace.com), ClubPenguin (http://www.clubpenguin.com), o Second Life (http://www.secondlife.com)e o OpenSim (http://opensimulator.org). (Giraffa, 2009). 


\subsection{Second Life}

O Second Life (SL) é atualmente um dos ambientes de realidade virtual mais conhecido no mundo. Com ambiente de visão tridimensional, foi tomado a princípio como um ambiente de entretenimento e interação social, mas chamou a atenção dos pesquisadores pelo crescente número de usuários, abrindo diversas possibilidades de exploração (Matos, 2011).

O SL foi lançado em 2003 pelo Linden Research INC, possui atualmente 16 milhões de usuários registrados, com grande capacidade de interação, configuração e de recursos técnicos. Uma das primeiras a utilizar os recursos deste ambiente com propósitos educacionais foi a IBM, uma das empresas responsáveis por criá-lo. Atualmente, mais de 100 instituições de ensino possuem atividades dentro do ambiente (Second Life, 2011).

No segmento de mundos virtuais encontramos o projeto SLoodle que possibilita o acesso a alguns recursos do ambiente Moodle diretamente do ambiente SL. É possível, por exemplo, estabelecer um chat entre os dois sistemas, ou mesmo adicionar textos a sua página do Moodle utilizando apenas o SL (Giraffa, 2009). Também há a possibilidade de acessar a página do Moodle utilizando o browser de acesso à Internet do SL (Giraffa, 2009). O projeto pode ser visitado no endereço http://www.sloodle.org/moodle/.

\subsection{OpenSim}

OpenSim faz parte do Projeto OpenSimulator (OS), é um servidor de Mundos Virtuais que pode ser utilizado para criar e desenvolver Ambientes Virtuais em 3D. Ele vem sendo desenvolvido por vários programadores. Pronto para uso, o OpenSimulator pode ser utilizado para criar um ambiente semelhante ao Second Life. Ele também pode ser facilmente estendido para produzir aplicações interativas em 3D mais especializadas. A interação acontece através de bate-papos e observação das ações de outros Avatares (http://opensimulator.org).

OpenSim é uma solução de código aberto, o que oferece alta compatibilidade com SL e com protocolos de comunicação com outras plataformas. É amplamente utilizado por universidades e grandes empresas (por exemplo, IBM, Microsoft). Sendo uma fonte aberta, o aplicativo concede aos designers a liberdade de criação de uma multiplicidade de interfaces de usuário, simulações e ambientes. Além disso, é especialmente adequado para a pesquisa, porque através da criação de um servidor privado, podemos ter experiências sem influência externa de outros. Em adicionalmente, o servidor de SL oficial restringe o número de objetos e o tamanho de scripts, dependendo do tamanho da área do terras de propriedade. Em OpenSim não existem tais restrições (Medeiros, 2005).

\section{Trabalhos Correlatos}

$\mathrm{Na}$ literatura foram encontrados trabalhos realizados utilizando mundos virtuais tridimensionais para reunir pessoas com objetivos em comum, mas já foram encontradas também algumas experiências pedagógicas que seguem:

- NMC: o NMC (New Media Consortium) é um consórcio de mais de 260 organizações focadas em aprendizado, que busca explorar e usar novas mídias e 
novas tecnologias. Dentre as instituições, distribuídas pelos Estados Unidos, Canadá, Europa, Ásia e Austrália, há universidades (Harvard, MIT, Stanford, UC Berkeley, UCLA, Illinois at Urbana-Champaign, Yale, etc.), museus, centros de pesquisa, fundações e empresas. O consórcio decidiu experimentar em mundos virtuais, por enxergar nesses ambientes um futuro para a educação, e depois de uma análise criteriosa, escolheu o SL como plataforma (Mattar, 2008). Hoje, o NMC tem uma das presenças mais marcantes em educação no SL, realizando uma série de atividades pedagógicas. $\mathrm{O}$ projeto pode ser visitado em http://www.nmc.org.

- Universidade do Porto: é uma instituição de ensino portuguesa, localizada na cidade de Porto que utiliza o SL criativamente para aulas, reuniões, exposição de trabalhos dos alunos e concursos.

- Unisinos: a Unisinos (RS) tem uma interessante e fundamentada experiência com o Grupo de Pesquisa: Educação Digital Unisinos/CNPq, não apenas no SL, mas no uso de mundos virtuais em geral, em educação. O grupo já utilizava, mesmo antes do surgimento do SL, outra ferramenta: o Active World. No primeiro semestre de 2008, o grupo da Unisinos construiu no SL a ilha Ricesu, que reúne diversas instituições católicas de ensino superior (Mattar, 2008).

Também foram encontrados trabalhos que buscam identificar e analisar quais são os benefícios da utilização dos AVAs tridimensionais, como é o caso da pesquisa de Andreas el. al. em 2010, que busca resultados na utilização do AVA tridimensional SLoodle para a aprendizagem colaborativa, e de Guomin e Jianxin também em 2010, que realizou uma pesquisa com objetivo de analisar o valor educativo do AVA SLoodle, mostrando as vantagens e os desafios encontrados.

\section{Metodologia}

A pesquisa está sendo realizada dentro do Grupo Teias - UFRGS juntamente com o Instituto Federal Farroupilha - Campus Panambi, estando esta pesquisa enquadrada como Pesquisa Qualitativa e de Desenvolvimento.

Na primeira etapa foi realizado um estudo dos diferentes ambientes imersivos existente atualmente para que fosse selecionado o que melhor se adaptaria a pesquisa. Nesta fase também foi realizada uma pesquisa bibliográfica sobre autores que pudessem embasar teoricamente a utilização desses ambientes como ambientes de ensino e aprendizagem, entre os autores estudos estão Levy Vigotisky e Paulo Freire.

Traduzindo para a atualidade trechos descritos por Vigotsky, pode-se notar a real importância da utilização de ambientes tecnológicos no desenvolvimento cognitivo. Engels e Vygotsky criticam os psicólogos e filósofos que sustentavam "que apenas a natureza afeta o homem e apenas as condições naturais determinam o desenvolvimento histórico do homem, enfatizando que ao longo da historia o homem também "afeta a natureza", transformando-a, criando para si novas condições naturais de existência." Além disso, Vigotsky argumentou que o efeito do uso de instrumentos sobre os homens é fundamental não apenas porque os ajuda a se relacionarem mais eficazmente com seu ambiente como também devido aos importantes efeitos que o uso de instrumentos tem sobre as relações internas e funcionais no interior do cérebro humano. (Vigotsky 1991). 
Paulo Freire adaptou seus métodos educacionais ao contexto histórico e cultural de cada aluno, possibilitando a combinação de seus conceitos "espontâneos" (baseados na pratica social), com os conceitos introduzidos pelos professores na situação de instrução. (Vygotsky 1991).

Através destes conceitos estabelecidos pelos autores, pode-se ter certeza de que a área de realidade virtual e os ambientes imersivos podem auxiliar indivíduos quanto ao seu desenvolvimento cognitivo, pois se tornam um meio adicional de aprendizado que se utiliza do meio social do qual os indivíduos estão amplamente envolvidos: os ambientes tecnológicos.

Na segunda etapa foi preparada a ilha para ser disponibilizada como ambiente de ensino e aprendizagem. Durante esta fase foi selecionado o OpenSim 0.7.4 como simulador de mudo virtual e também o Firestorm versão 4.0.1 como visualizador do mundo virtual. A escolha do Opensim se deu pelo fato de ser livre, não trazendo custos adicionais para o projeto e o Firestorm foi selecionado pelo fato de permitir a importação de objetos modelados em outros ambientes de desenvolvimento, através da criação de arquivos com extensão dae. Foram utilizados os modeladores Sketchup na versão 7 e o Autodesk 3DsMax versão free para estudantes para desenvolvimento da "mundo". Pretende-se futuramente testar o modelador Blender $2.63^{\mathrm{a}}$ para modelagem dos objetos internos que ainda não foram desenvolvidos.

Durante a realização desta etapa foram encontrados alguns problemas no desenvolvimento, como avatares que perpassavam objetos desenvolvidos e também problemas com a importação entre o software de desenvolvimento e o ambiente imersivo. Notou-se que muitos objetos disponíveis em sites de forma gratuita, não se comportavam da maneira esperada e optou-se pela modelagem completa tanto do prédio do IF Farroupilha como dos objetos que serão colocados no seu interior.

$\mathrm{Na}$ etapa atual de desenvolvimento, o grupo está realizando a modelagem dos últimos objetos para o interior do ambiente, fazendo a implantação do ambiente no servidor e preparando os materiais didáticos para as aulas que serão ministradas neste ambiente. Também se pretende futuramente a utilização do Sloodle em conjunto com o mundo virtual para disponibilização dos materiais das disciplinas de forma mais facilitadas para os professores.

\section{VirtualTche}

O VirtualTche é um grupo de estudos formado/composto por professores e alunos da Universidade Federal do Rio Grande do Sul e do Instituto Federal Farroupilha - Campus Panambi e busca o desenvolvimento de formas de trabalho onde os alunos possam fazer a interação e adquirir conhecimentos de uma forma mais natural e dinâmica do que a disponibilizada pelos tradicionais ambientes virtuais de aprendizagem. Essa interação pretendida fará com que os alunos possam trocar informações sobre os conteúdos das aulas com professores e com outros estudantes, bem como comunicar-se em um ambiente imersivo e totalmente voltado para conteúdos educacionais.

O início dos trabalhos deu-se através do conhecimento e dos estudos sobre ambientes imersivos já utilizados por outras universidades e empresas. A opção de desenvolver o "mundo" VirtualTche através da utilização do OpenSim se deu pelas condições de acesso e manipulações que o ambiente dispõe até o momento, sendo uma 
plataforma estável e com condições de abrigar o mundo do Instituto Federal Farroupilha - Campus Panambi, fornecendo acesso a um grupo de estudantes que futuramente utilizarão este mundo como uma sala de aula virtual.

A área física do instituto dispõe atualmente de dois prédios, um prédio alocado com salas de professores, biblioteca e outros setores burocráticos e outro prédio destinado ao laboratório de química, física e alimentos, os laboratórios de informática e as salas de aula, conforme figura 1.

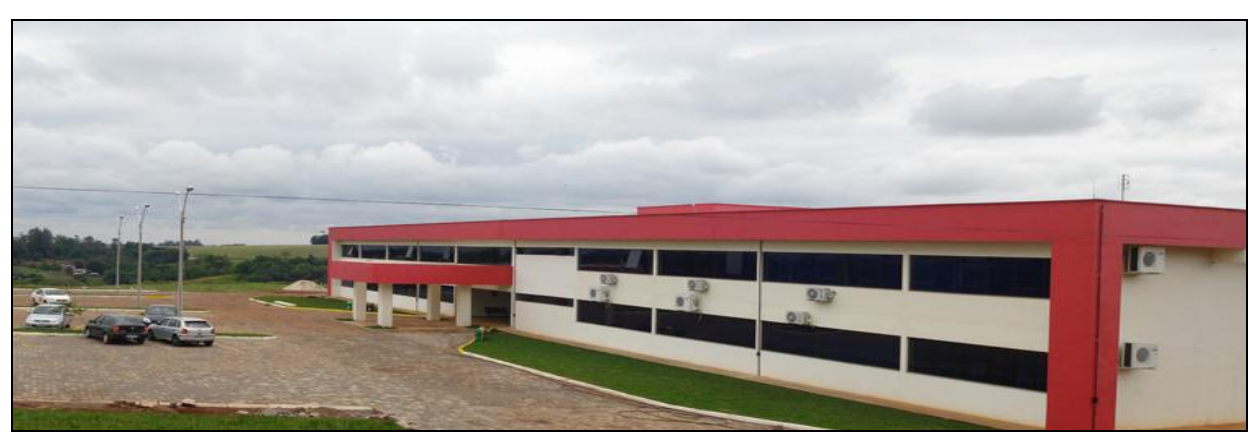

Figura 1. Foto do Campus Instituto Federal Farroupilha - Campus Panambi

A figura 2 apresenta a imagem obtida através do visualizar Firestorm modelada e após importada para o OpenSim, onde o avatar está visitando o mundo desenvolvido IF Farroupilha.

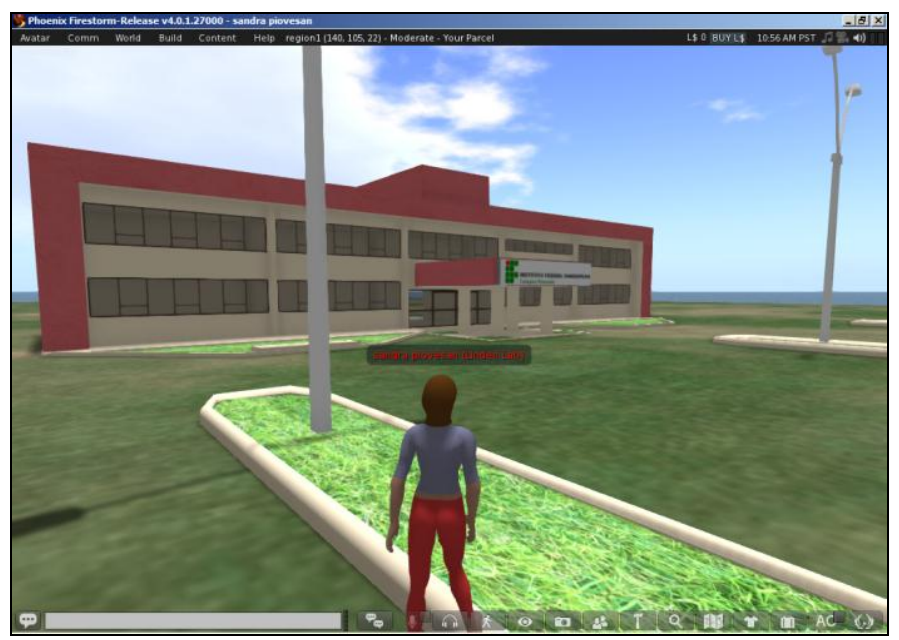

Figura 2. IF Farroupilha

Os objetos a serem desenvolvidos, de acordo com a real distribuição deles dentro do Instituto ainda é uma etapa a ser vencida. A figura 3 apresenta o hall de entrada do IF Farroupilha já desenvolvida. 


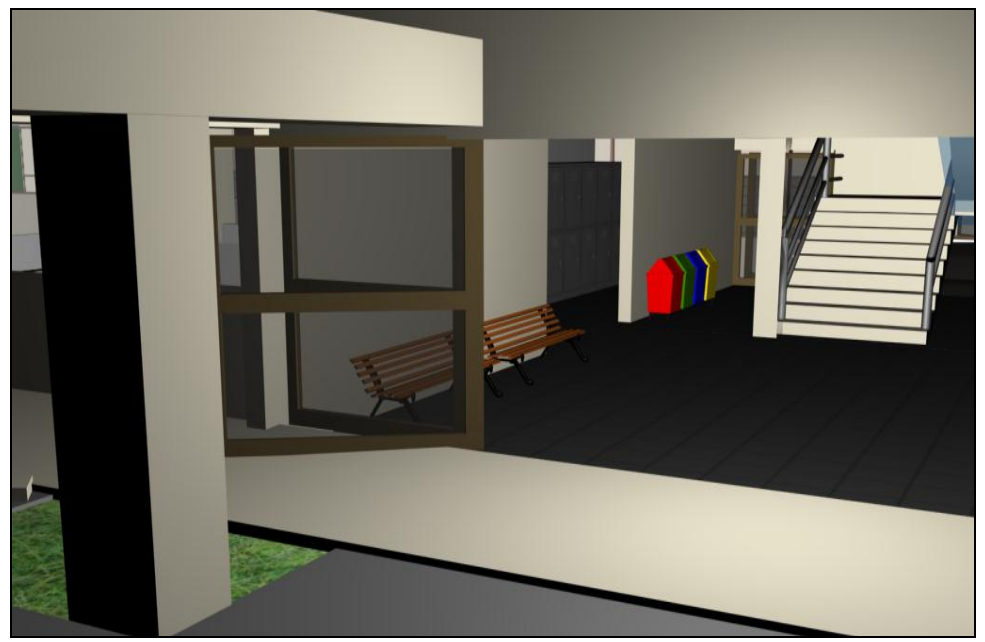

Figura 3. Hall de Entrada

\section{Considerações Finais}

Tudo começou através dos interesses simultâneos pela área de realidade virtual unida ao ambiente escolar, onde verificamos a possibilidades de desenvolvimento pessoal e de aprendizagem dos alunos. Desta forma, os trabalhos e os esforços voltaram-se para o desenvolvimento de um ambiente escolar que possibilite a interação e o desenvolvimento dos alunos, visto que é através da interação com o meio social que se dá o desenvolvimento pessoal.

A utilização do ambiente virtual de aprendizagem Moodle faz parte da vida diária de alunos e professores, onde estes utilizam este espaço para apoiar as aulas, disponibilizar materiais e possibilitar a troca de informações em horários extraclasse. A utilização de um ambiente de realidade virtual, totalmente imersivo, substituindo este tradicional ambiente virá a apoiar as práticas existentes hoje na instituição e possibilitar outras formas de integração de indivíduos com a tecnologia.

\section{Referências}

Andreas, K.; Thrasyvoulos, T.; Stavros, D.; Andreas, P.; Collaborative Learning in OpenSim by Utilizing SLoodle. In: 2010 Sixth Advanced International Conference on Telecommnications, IEEE, 2010.

Beyer, H.; Inclusão e Avaliação na escola: de alunos com necessidades especiais. Porto Alegre: Mediação, 2005.

Braga, Mariluci. Realidade Virtual e Educação. Revista de Biologia e Ciências da Terra, 2001. Acesso em : http://eduep.uepb.edu.br/rbct/sumarios/pdf/realidadevirtual.pdf, 2011

Gadia, C., A.; Tuchman, R.; Rotta, N., T.; Autismo e doenças invasivas de desenvolvimento. Jornal de Pediatria. Acesso em: www.scielo.br/pdf/jped/v80n2s0/v80n2Sa10.pdf, 2011. 
Giraffa, L., M., M.; Uma odisséia no ciberespaço: O software educacional dos tutoriais aos mundos virtuais. RBIE, 2009.

Guomin, Z.; Jianxin, Z.; Na Education Value Analysis of SLoodle-based Distribuidet Virtual Learning System. In: Second International Workshop on Education Technology and Computer Science, IEEE, 2010.

H. Dohi and M. Ishizuka, "A Life-Like Agent Interface System with Second Life Avatars on the OpenSimulator Server", A.A. Ozok and P. Zaphiris (Eds.): Online Communities, LNCS 5621, pp. 182-190, 2009. Springer-Verlag Berlin Heidelberg.

Hill E., L. ; Frith U.; Understanding autism: insights from mind and brain. Philos Trans R Soc Lond B Biol Sci. 2003;358(1430):281-9.

Jordan R.; Powell, S; Understanding and Teaching Children with Autism. West Sussex, England: John Wiley\&Sons, 1995.

Mattar, J.; O Uso do Second Life como ambiente de Aprendizagem. Revista Fonte, 2008.

Matos, E., S.; A Virtualidade real: Possibilidades de Aplicação de Realidade Virtual na Educação Mediada por Computador. Anais SBC, 2008. Acesso em : http://www.prodepa.gov.br/sbc2008/anais/pdf/arq0169.pdf, 2011.

Medeiros, Maria Beatriz; Amadeu, Flávia. Immersive and Participative Environments. ReVISta. Arte e Conhecimento. Ano 4, n. 4 (set 2005). Brasília: IdA, 2005, p-159171; ISSN 1518-5494.

Minshew N., J.; Payton J., B.; New perspectives in autism, Part I: the clinical spectrum of autism. Curr Probl Pediatr. 1988;18:561-610.

Passerino, L., M; Avila, B., G.; Bez, M., R.; Scala: um Sistema de Comunicação Alternativa para o Letramento de Pessoas com Autismo. Cinted-UFRGS, 2010.

Passerino, L., M.; Santarosa, L., M., C.; Possibilidades da Mediação Tecnológica na Inclusão Escolar de Autistas. Anais do XXVI Congresso da SBC, 2006.

Rapin I; Autistic children: diagnosis and clinical features. Pediatrics. 1991;87:751-60.

Santaella, Lucia. Cultura e artes do pós-humano, São Paulo, Paulus, 2003

Second life. Acesso em: http://secondlife.com/, 2011.

Vila, C.; Diogo, S.; Sequeira, S.; Autismo e Síndorme de Asperger. O portal dos Psicologis. Acesso em: <http://www.psicologia.com.pt>, 2011

Vigotsky, L. S. A formação social da mente: o desenvolvimento dos processos psicológicos superiores. 7 ed. São Paulo: Martins Fontes, 1991. 